\title{
NON-DISJUNCTION OF THE SEX CHROMOSOMES OF DROSOPHILA
}

\author{
CALVIN B. BRIDGES \\ The Zoölogical Laboratory, Columbia University
}

A certain kind of exception that has come up during the course of my work on Drosophila ampelophila, with Dr. T. H. Morgan, bears directly upon the problem of sex determination, and especially upon the view that the chromosomes are the carriers of the differentiators of the hereditary characters. The evidence which I am to present deals with the $X$-chromosome ${ }^{1}$ of Drosophila, concerning which the evidence relating to sex linkage and the linear arrangement of factors is more definite and complete than in any other case. Ordinarily, when a female with white eyes is mated to a wild male with red eyes, the daughters have red, and the sons, white eyes. This is a typical case of the crisscross inheritance characteristic of sex linkage. The cytological evidence of Miss Stevens shows that Drosophila belongs to that group of forms in which the female has two $X$-chromosomes and the male an unpaired $X$-chromosome. In spermatogenesis, half of the spermatozoa receive this $X$ and half do not. In oögenesis, each gamete receives one $X$. The fertilization of any egg by a one- $X$ sperm, results in a female $(X X)$, while any egg fertilized by a no- $X$ sperm results in a male $(X-)$. Morgan has explained the case of criss-cross inheritance on the ground that the $X$ chromosome is the carrier of all sex linked factors. The sons are matroclinous because they receive their unpaired sex chromosome directly from the mother, and must show all the sex linked characters which she showed. Each daughter, of the case cited, is red because the paternal of her two sex chromosomes bears the dominant red, and the maternal bears the recessive white.

${ }^{1}$ I have used ' $X$,' ' $X$-chromosome' and, sex-chromosome' interchangeably. 
In the exceptional case that I have found, where the mating was like that just described, about 5 per cent of the daughters are like the mother and 5 per cent of the sons are like the father.

This anomolous result may be explained, if, in such mothers, a certain per cent of maturations are of a type characterized by non-disjunction, ie., eggs are formed which contain two sex chromosomes instead of the normal one, and other eggs corresponding to them contain no sex chromosome. If in one of the two maturation divisions of the egg, the two sex chromosomes that usually pass to opposite poles, one going out in the polar body and the other remaining in the egg, should sometimes not disjoin from each other, but should lag upon the spindle so that both pass into the polar body or the egg, then there would result eggs with two chromosomes and other eggs with no sex chromosome. The eggs of such a female will then be of three classes, namely, a large normal class with one sex chromosome, and two small equal classes containing respectively, two and no sex chromosomes.

If sex linked characters are determined by factors carried in definite loci in the sex chromosome, then we have a way of tracing the maternal and paternal chromosomes. For example, all the $X$-chromosomes of a white female, which produces the three kinds of eggs above, are white bearing. Any $X$-chromosome of the wild male, to which she is mated, is red bearing.

The results of this cross are summarized in the diagram below, ${ }^{2}$ and are as follows:

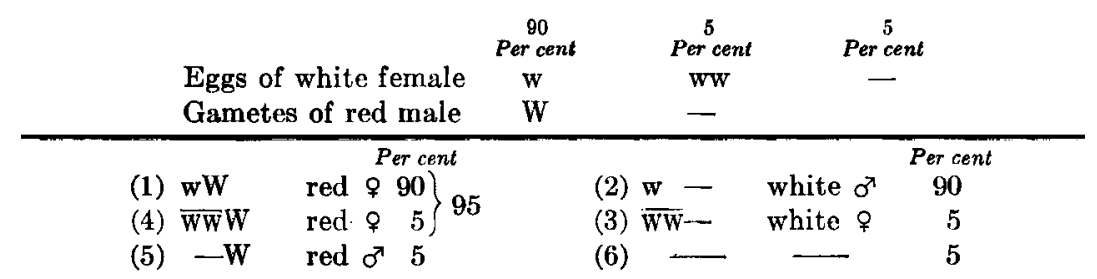

(1) The one- $X$ egg fertilized by the one- $X$ sperm will give a female $(X X)$, red, heterozygous for white.

(2) The one- $X$ egg

${ }^{2}$ In the diagram the $w$ indicates the sex chromosome which bears white, and the $W$ the red bearing chromosome. The dash represents the no- $X$ gamete. 
by the no- $X$ sperm will give a male $(X-)$, whose only $X$ came from his mother, and hence determines him as white. The one$X$ eggs give, then, the normal criss-cross result characteristic of sex linked inheritance, (white $\circ \times$ red $\sigma^{7}=$ red $q+$ white $\left.\sigma^{7}\right)$. The two- $X$ egg will give females only, since it is already duplex with respect to the sex differentiator. (3) If this egg is fertilized by a no- $X$ sperm, there should result a female $(X X)$, both of whose sex chromosomes are maternal, and would thus produce white. (4) The two- $X$ egg, fertilized by the one- $X$ sperm, will produce an individual with three allelomorphic sex chromosomes, two of which are white, and the other red bearing. This fly will, if viable, be a red female, indistinguishable from the large class above. (5) The no- $X$ egg by the one- $X$ sperm will produce a male $(X-)$, differing from the normal male in that his $X$ is not maternal but paternal, and would produce a red fly. (6) The no- $X$ egg by the no- $X$ sperm will produce a zygote with no $X$, and such a zygote, I believe, is not viable.

The presence of the white gene in the maternal chromosomes gives us a means of distinguishing between all the classes, except between the three- $X$ and the heteroxygous red $X X$ types.

Non-disjunction of the other chromosomes (autosomes) has not been observed in these crosses.

If 10 per cent of the eggs of such a female matured in a nondisjunctional manner the proportions of the $F_{1}$ would be:
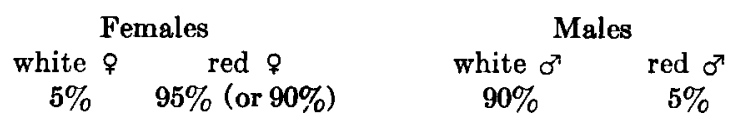

The evidence in support of these conclusions is as follows. From time to time in an $F_{1}$ where sex-linked characters were concerned, females or males have arisen which I was unable to explain on any current Mendelian hypothesis. Some of these I bred, and the offspring were as hard to explain as the original exceptions. One of the first cases was the following. I crossed a recessive pink female, of a stock which had been pure for some twenty-five generations, to a male which was a triple recessive, namely, white miniature black. The normal $F_{1}$ expectation for 
this cross is red females and males. Of the factors concerned, pink of the female is in the third chromosome and black of the male is in the second chromosome. Both white and miniature are in the sex chromosome at a distance from each other of approximately 32 units (Sturtevant, '13 a). The normal $F_{1}$ female, then, is heterozygous for four factors, white, miniature, black, and pink. The $F_{1}$ male is heterozygous for black and pink, but is normal with respect to white and miniature, since his only sex chromosome came from his mother, who was neither white nor miniature. I had three cultures of $F_{1}$ and they produced in aggregate the expected red females and males to the number of 251 and 305 respectively. But I found also three white miniature males, one in each culture. Their fathers were white and miniature, but these were not $P_{1}$ males left over in the culture, because they were very young (young flies are very light in body color and show other characteristic differences from the adult), and occurred after I had emptied the culture bottles several times. Fortunately I bred one of these males to virgin sisters, which were heterozygous for white, miniature, black, and pink. The results showed conclusively that the male had really come from the cross and was not the result of any contamination, in that it gave in the next generation all expected classes from the cross of an $F_{1}$ female by a white miniature male heterozygous for black and pink, that is, there appeared blacks and pinks, males and females, whites and miniatures, in all the combinations and permutations expected.

Since my interest was centered primarily in the white and pink characters, and I did not at that time realize the importance of the problem, I counted the offspring of the non-disjunctional male only with respect to white and pink, although the occurrence of blacks and miniatures of both sexes was noted. ${ }^{3}$ The count approximated expectation: $(4: 3: 1: 4: 3: 1)$.

\begin{tabular}{ccccccc} 
white $\&(4)$ & red $\&(3)$ & pink $\&(1)$ & white $\sigma^{7}(4)$ & red $\sigma^{7}(3)$ & pink $\&(1)$ \\
106 & 93 & 30 & 111 & 78 & 30 \\
\hline
\end{tabular}

${ }^{3}$ For comparison $I$ give the $\mathrm{F}_{2}$ from normal males by sisters such as those to which I mated the patroclinous male. $\begin{array}{ccccc}\text { red } \&(6) & \text { pink } \&(2) & \text { white or (4) } & \text { red } \sigma^{7}(3) & \text { pink or }(1) \\ 733 & 196 & 354 & 340 & 75\end{array}$ 
There is no question of association here, for the white and pink are in different chromosomes, the sex and the third.

The interpretation of these results is as follows: among the $P_{1}$ pink females used there was at least one female which formed some eggs in a non-disjunctional manner with respect to the sex chromosome, but not with respect to the second and third chromosomes. The duplex sex chromosome of the pink female contained only non-mutated genes. ${ }^{4}$ Wherefore the female resulting from the fertilization of the two- $X$ egg would be indistinguishable from the other $F_{1}$ females, irrespective of the character of the sperm. But the no- $X$ egg, if met by a one- $X$ sperm would produce a male exhibiting all the sex chromosomal characters of the $\mathrm{P}_{1}$ male which supplied the chromosome. Since the $\mathrm{P}_{1}$ male was white and miniature, these characters were the ones which showed in the three such males of this experiment.

If this non-disjunction involved the second chromosome, then in $F_{1}$ there might appear some black females and males, which was not the case. The white miniature males, moreover, should be black but simplex, and in the next generation there should result a great overproduction of blacks, which was not observed. A similar disturbance in the reduction of the third chromosome would have disturbed the $F_{2}$ ratio from that observed.

The case just cited illustrates the production and composition of the patroclinous males. The next case gives evidence upon these points for the females. I crossed some white pink females to some eosin vermilion pink males. The expectation in $F_{1}$ is white pink males and white-eosin compound pink females heterozygous for vermilion. This cross is reported in this number and an analysis given by Morgan and Bridges. Of the factors concerned, the third chromosomal pink entered the zygote from both sides so that all flies in this experiment are pink. White and vermilion are sex chromosomal and so in expectation all the sons of the white not-vermilion mother are white and likewise notvermilion. White and eosin are allelomorphic to each other, that is, they occupy the same locus in the sex chromosome. Of the two homologous sex chromosomes of the $F_{1}$ female the mater-

Except the gene for non-disjunction itself, as will appear later. 
nally derived carried white and the paternally derived carried eosin. The peculiarity of this combination in the female is that in color it looks like an intermediate between white and eosin, hence the name white-eosin compound. Several cultures of the type described above produced in aggregate 2073 white-eosin compound pink females heterozygous for vermilion, and 2037 white pink males. But in addition there were nine, white females and two males of a grade of color corresponding to eosin vermilion pink, that is, to the color of the fathers. These two males were sterile so I could not test the correctness of my classification.

With the matroclinous females however, I made thefollowing tests. One of them I mated to her white brothers, and in the next generation got 51 white females and 60 white males. This test only shows that my classification of the female as white was correct, for she gave no eosin sons, as did her ordinary sisters mated to the same males.

Another of these white females I mated to a double recessive male, namely vermilion pink. All the ordinary sisters were heterozygous for vermilion, but if my hypothesis is correct, then the non-disjunctional female should be the exact counterpart of her mother not only in being white, (as the first test showed she was), but also in being pure for not-vermilion, although her father was vermilion. If she were heterozygous for vermilion, it would show in that half of her sons and daughters would be vermilion. As a matter of fact none of her daughters were vermilion and all of her sons were white, with one exception. She was, then, the exact counterpart of her mother as far as all sex chromosomal characters were concerned. The one exception, a vermilion pink male, was likewise an exact counterpart of his father. The actual offspring of the second white female by the vermilion pink male of unrelated stock were:

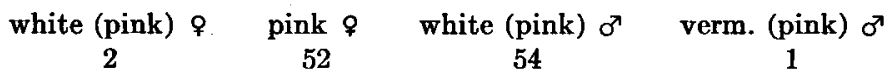

All the flies are pink because pink entered from both sides. The presence of the one vermilion pink male and the two white 
pink females brings out a new point, namely, that a non-disjunctionally produced female manifests her peculiarity directly in $\mathbf{F}_{1}$ by producing more like herself. The two white females produced here came from the union of the two- $X$ egg with the no- $X$ sperm of the vermillon pink male, and should be entirely maternal and entirely non-paternal in sex chromosomal composition, i.e., they should be exact counterparts once more of their mother. Wherefore, if they should be mated to a male like their father, the same story should be repeated. This test was made:

$P_{1} \quad$ white pink matroclinous $\& \times$ vermilion pink (outcross) $\sigma^{7}$

$F_{1} \quad$ white (pink) $\%$ pink $\%$ white (pink) $\sigma^{\top}$ verm. pink $\sigma^{\top}$

The culture unfortunately ran out before the point was completely established, but the appearance of the one patroclinous vermilion pink male makes it certain that white pink females would ultimately have appeared.

The other of these two white pink females (the first was mated to the vermilion pink or) was mated to an eosin male of an unrelated stock. The story was repeated, but with a very important modification, namely, that the non-disjunctional males were eosin. The results were as follows:

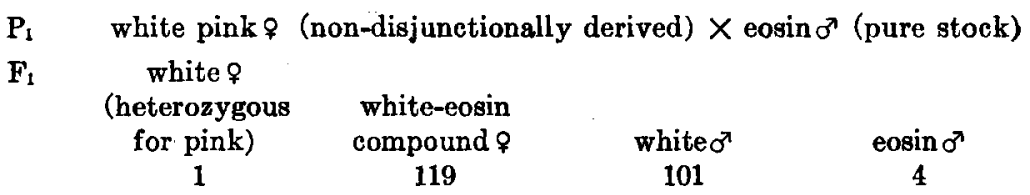

The results show that the non-disjunctionally derived female produced non-disjunctional females once more, and that like her mother she was homozygous for white (all female offspring whiteeosin compound and all normal sons white), and that the sex chromosomal characters of the non-disjunctional sons do not depend in any degree upon the composition of their mother but only upon that of their father, in the first case cited, white miniature, here eosin. Moreover, of sister females, one produced vermilion and the other eosin patroclinous sons when mated to 
vermilion and eosin males respectively. Thus for four sex chromosomal factors the same conclusions hold.

Since it has been shown that the non-disjunctionally produced female hands her peculiarity on directly to her $F_{1}$ non-disjunctional daughters, we can test the non-disjunctional sons in that respect. The offspring of the white miniature exceptional male give us no information on the question, because the classes of offspring which would show the effect can not be separated from the large normal class already present. The vermilion pink male, however, I mated to wild females. The first generation consisted of red females 223 and red males 159 and no non-disjunctionals. $F_{2}$ was like that from a normal stock vermilion pink male, namely:

$$
\begin{array}{cccccc}
\text { red } \% . & \text { pink } \% & \text { red } \sigma^{7} & \text { vermilion } \Im^{7} & \text { pink } \sigma^{7} & \text { vermilion pink } \sigma^{7} \\
45 & 22 & 27 & 27 & 6 & 9
\end{array}
$$

The fact that the non-disjunctional female gives non-disjunctionals in $F_{1}$ is the result one would expect if the cause of this peculiar oögenesis were itself a sex chromosomalgene. If such a gene were present in each of the sex chromosomes of the original female all her exceptional daughters would of course have the same condition and thus the line would be perpetuated parthenogenetically, so to speak, indefinitely. But with regard to the exceptional male it should be otherwise, for he gets no sex chromosome from his mother and consequently can neither have nor transmit the non-disjunction gene.

Before passing to the next instances, I can present some evidence on the composition of the normal pink females and white males produced in the cross of the white pink non-disjunctional female to the vermilion pink male (out-cross). A few of the normal pink females and white males produced:

$$
\begin{array}{ccccc}
\text { pink } q(4) & \text { white } q(4) & \text { white } \sigma^{7}(4) & \text { verm. pinko } \sigma^{7}(3) & \text { pink o }(1) \\
74 & 77 & 66 & 62 & 15
\end{array}
$$

The simple ratio is altered somewhat by association of white and vermilion, but, is otherwise a wholly normal result. 
Two other white pink females (of the nine) I mated separately to wild males. They produced:

$\begin{array}{cccc}\text { white } \% & \text { red } \% & \text { white } \sigma^{7} & \text { red o⿱ } \\ 7 & 192 & 131 & 8 \\ 5 & 151 & 147 & 11 \\ - & - & - & - \\ 12 & 343 & 278 & 19\end{array}$

These two instances illustrate once more the fact that the sex chromosomal character of the non-disjunctional female depends solely upon the sex chromosomal character of her mother and in nowise upon that of her father. But with the non-disjunctionally produced male the result is exactly reversed, for each time the character of the non-disjunctional male depends not upon the characteristics of the mother (other than non-disjunction itself) but is a duplicate of that of his father, in this case completely normal.

If the third chromosome is not involved in this non-disjunction then we should expect that as far as the distribution of pink is concerned, the results would be exactly that of a normal cross, that is, both the non-disjunctional females and males should be heterozygous for pink, because their mother was homozygous for pink and their father was not-pink. To test this point further I crossed three non-disjunctionally produced females (of the twelve) to non-disjunctionally produced males (of the nineteen). The next generation was:

$\begin{array}{cccccc}\text { white } q & \text { red } q(3) & \operatorname{pink} q(1) & \text { white } \sigma^{7}(4)^{\circ} & \text { redo } & \text { pinko } \\ 1 & 52 & 37 & 115 & 0 & 0 \\ 7 & 101 & 42 & 141 & 0 & 0 \\ - & - & - & - & - & - \\ 8 & 153 & 79 & 256 & 0 & 0\end{array}$

One can see here in the same cross both non-disjunction and normal segregation, but with respect to different chromosomes. The non-disjunction results are, the sons all white to correspond to the composition of their mother, and the occurrence of the white non-disjunctional females. The production of pink females shows that both parents were heterozygous for pink, since if 
either one were pure for not-pink, (as in the next case below) no pinks would have appeared in the offspring. Had the numbers been large enough, exceptional males should have appeared in the proportion of three red to one pink among themselves.

A second non-disjunctional white female (of the twelve) I crossed to a wild male and obtained:

$\begin{array}{cccc}\text { white } 9 & \text { red } \sigma^{\top} & \text { white o } & \text { red } \sigma^{\top} \\ 15 & 159 & 146 & 13\end{array}$

Here no pink appeared although the cross was externally the same as the previous one. The interpretation is that the male in the first case differed from the wild male in that it was heterozygous for pink.

To effectually show that the non-disjunctional males were heterozygous for pink I mated four (of the nineteen) to stock pink females, and as I expected, half of the females and half of the males were pink:

$\begin{array}{cccc}\text { red } & \text { pink } & \text { red } \sigma^{\top} & \text { pink } \sigma^{\top} \\ 70 & 69 & 63 & 62\end{array}$

To show, once more, that it was the female only that was responsible for the production of exceptions in $F_{1} I$ simulated the cross of non-disjunctional white pink female by non-disjunctional male given above by testing a non-disjunctional brother of the first by a white female of another stock. The result was simply red females 105 and white males 74 , with no exceptions.

A third male (of the nineteen) I tested to an eosin female with still no exceptions appearing in $F_{1}$ among the 143 red females and 146 eosin males.

There still remained to be tested only the normally produced females (343) and males (278). Two pairs from these gave the ordinary Mendelian result:

$\begin{array}{cccccc}\text { white } \%(4) & \operatorname{red} \%(3) & \operatorname{pink} \text { \% (1) } & \text { white } \sigma^{7}(4) & \text { red or(3) } & \text { pink } \sigma^{7}(1) \\ 52 & 43 & 15 & 59 & 44 & 23 \\ 45 & 37 & 15 & 48 & 29 & 12 \\ -57 & - & - & - & - & - \\ 97 & 80 & 30 & 107 & 73 & 35\end{array}$


I continued tests of this line of females for three generations more as follows: From the fifteen non-disjunctional females I selected three which I mated to four red brothers. If both the females and males were heterozygous for pink I should get pink females among the offspring and these in fact I found as follows:

$\begin{array}{ccccc}\text { white } \% & \text { red } \% & \text { pink } \% & \text { white } \sigma^{7} & \text { red } \sigma^{7} \\ 4 & 99 & 17 & 134 & 6^{*} \\ 10 & 158 & 29 & 173 & 11 \\ \frac{1}{14} & -257 & -46 & -307 & 17\end{array}$

*In addition to these red males there was a red eyed lateral gynandromorph, which is being figured by Morgan in "Heredity and Sex."

From among these fourteen matroclinous white females I mated two to four of their red brothers. The results were:

$\begin{array}{ccccc}\text { white } \% & \text { pink } \% & \text { red } \% & \text { white } \sigma^{7} & \text { red } \sigma^{7} \\ 9 & 17 & 134 & 95 & 5\end{array}$

It is quite probable that only one of these flies was heterozygous for pink, since theoretically, only half of the fourteen flies should be heterozygous for pink. For they came from a cross of heterozygous non-disjunctional female to a wild male (not-pink).

The five cases like the last sum up to:

$$
\begin{array}{cccccc}
\text { white } \% & \text { pink } \% & \text { red } \% & \text { white } \sigma^{\top} & \text { red } \sigma^{\lambda} & \text { pink } \sigma^{7} \\
22 & 142 & 544 & 658 & 22 & 0
\end{array}
$$

Expectation calls for fifteen red to seven pink among the exceptional males. I think that probably the non-appearance of the pink males has no significance.

Three more of the fourteen white females I now mated to red males of wild stock. The results were

$\begin{array}{cccc}\text { white } 9 & \text { red } \% & \text { white } & \text { red } \\ 15 & 132 & 95 & 9 \\ 4 & 98 & 80 & 0 \\ \overline{19} & -230 & \overline{175} & 9\end{array}$

From the nineteen exceptional white females here produced, I mated three to wild males and obtained: 


$\begin{array}{cccc}\text { white } \% & \text { red } \% & \text { white } \sigma^{7} & \text { red } \sigma^{7} \\ 2 & 128 & 86 & 2 \\ 3 & 131 & 157 & 2 \\ - & - & - & - \\ 5 & 259 & 243 & 4\end{array}$

The cross of white pink females by eosin vermilion pink males was originally planned only on a small scale for the purpose of making observations on the eye color of the daughters. When there appeared a few matroclinous daughters, the scale of the experiment was increased. However, the color of the males which would correspond to the exceptional females is very close to white, and it would be easy to miss some of them among the normally produced whites. Accordingly, some of the females from the white pink stock were mated to wild males. I raised four cultures, using as the mothers in each case five white pink females. The results show that non-disjunction was wide-spread in that stock and had maintained itself there during the six months (some fifteen generations) between the two crosses. The results are tabulated below:

$P_{1}$

\begin{tabular}{cccc}
\multicolumn{2}{c}{ white pink $\%$} & \multicolumn{2}{c}{ wild o } \\
white $\%$ & red $\%$ & white $\sigma^{7}$ & red $\sigma^{7}$ \\
7 & 212 & 207 & 6 \\
1 & 187 & 186 & 2 \\
1 & 191 & 247 & 3 \\
2 & 220 & 205 & 8 \\
$\overline{11}$ & - & - & - \\
\hline 1 & 910 & 845 & 19
\end{tabular}

That it is the same phenomenon here is shown by the results of mating a white daughter (of the seven) to wild males.

$P_{1}$

$$
\begin{array}{cccc}
\multicolumn{2}{c}{\text { white } \%} & \multicolumn{2}{c}{\text { wild } \sigma^{7}} \\
\text { white } \% & \text { red } \% & \text { white } \sigma^{7} & \text { red } \sigma^{7} \\
3 & 99 & 64 & 3
\end{array}
$$

Half of these white females should be heterozygous for pink if they really were produced in the manner assumed. One bred to a pink male showed that she was in fact heterozygous for pink.

$P_{1}$

$\mathbf{F}_{1}$

\begin{tabular}{ccccc} 
white (of the three) & \multicolumn{2}{c}{ pink $\sigma^{\pi}$} \\
white \& & red $\%$ & pink $\%$ & white $\sigma^{7}$ & red $\sigma^{7}$ \\
2 & 70 & 71 & 126 & 2
\end{tabular}


I have been able to detect non-disjunction in certain other stocks, notably eosin. Of the females tested, one gave the following offspring by a white miniature male.

$\begin{array}{ccccc}F_{1} & \text { eosin } q & \text { white-eos. comp. } \% & \text { eosin } \sigma^{7} \text { (long) } & \text { white min. } \sigma^{7} \\ & 7 & 154 & 134 & 2\end{array}$

These seven eosin females contained no trace of the paternal white and miniature, for one mated to a wild male gave no white or miniature sons, but only eosin not-miniature sons, and further patroclinous sons, which were of course red.

$\mathbf{P}_{\mathbf{1}}$ \begin{tabular}{cccc} 
eosin $\varphi$ (of the seven) & \multicolumn{2}{c}{ wild $\sigma^{\top}$} \\
$\operatorname{eosin} \varphi$ & red $\varphi$ & eosin $\sigma^{\top}$ & red $\sigma^{\top}$ \\
3 & 118 & 120 & 5
\end{tabular}

The purity of the eosin daughters is shown even more clearly by. back-crossing three other females (of the seven) to white miniature males. One would ordinarily expect half the males and half the females to be miniature and white, with the proportions showing the association of white and miniature. But no such effect was produced, as one can see in the progeny:

$\begin{array}{cccc}\operatorname{cosin} \subsetneq & \text { white-eos. comp. } \% & \text { eosin } \sigma^{\lambda} & \text { white min. } \sigma^{7} \\ 15 & 133 & 135 & 9\end{array}$

The white-eosin compound is a very efficient aid in studies such as these, since it enables one to tell at a glance the composition of the female whether pure eosin, pure white, or whiteeosin; determining in this case that the thirteen daughters were pure eosin and not-white, and that the 133 daughters were true hybrids, i.e., white-eosin compounds.

For the sake of maintaining stock, I have continued this line a little further by breeding one of the three eosin females to one of her red brothers.

\begin{tabular}{ccccc}
$P_{1}$ & \multicolumn{2}{c}{$\operatorname{eosin} \%$} & \multicolumn{2}{c}{ red $\sigma^{7}$} \\
$F_{1}$ & $\operatorname{eosin} \%$ & $\operatorname{red} \%$ & eosin $\sigma^{7}$ & red $\sigma^{7}$ \\
& 5 & 81 & 76 & 6
\end{tabular}

The next generation in the same manner was:

$\begin{array}{cccc}\text { eosin } \odot & \text { red } \odot & \text { eosin } \sigma^{7} & \text { red } \sigma^{7} \\ 8 & 132 & 139 & 14\end{array}$


Since it makes no difference what male is used, I paired two of the eight eosin females to white males and obtained.

$\begin{array}{cccc}\text { eosin } \subsetneq & \text { white-eos. comp. } \& & \text { eosin } \sigma^{7} & \text { white } \sigma^{\top} \\ 10 & 99 & 110 & 10 \\ 6^{*} & 91 & 93 & 14\end{array}$

* "In addition to these six eosin females there appeared a female with a penis. like that figured in "Heredity and sex." The eye color was eosin $\left(w^{e}-w^{e}\right)$, It is possible that this was a three chromosomal form, or else caused by somatic non-disjunction. Neither gynandromorph would breed.

I shall cite here several cases of non-disjunction which have appeared in other experiments, and which show that although the phenomenon is infrequent, it is wide-spread in diverse stocks

\begin{tabular}{|c|c|c|c|c|}
\hline $\begin{array}{l}P_{1} \\
F_{1}\end{array}$ & \multicolumn{2}{|r|}{ eosin 9 웅 } & \multicolumn{2}{|c|}{ white vermilion pink $\sigma^{7} \sigma^{7}$} \\
\hline$F_{1}$ & $\begin{array}{l}\operatorname{eosin} \varphi \\
2\end{array}$ & $\begin{array}{c}\text { white-eos. comp. } \% \\
273 \\
298^{*}\end{array}$ & $\begin{array}{l}\operatorname{eosin} \sigma^{7} \\
260 \\
277\end{array}$ & $\begin{array}{c}\text { white verm. } 0^{7} \\
2 \\
1\end{array}$ \\
\hline $\mathbf{P}_{1}$ & & white 우 우 & $\operatorname{eosin} v e$ & ilion pink $\sigma^{x} \sigma^{x}$ \\
\hline $\mathbf{F}_{1}$ & $\begin{array}{c}\text { white } \% \\
0\end{array}$ & $\begin{array}{c}\text { white-eos. comp. } 9 \\
151\end{array}$ & $\begin{array}{l}\text { white o' } \\
\quad 142\end{array}$ & $\begin{array}{c}\text { eos. verm. } 0^{x} \\
1\end{array}$ \\
\hline
\end{tabular}

* There appeared here one female with one eye white and one eye eosin. Her offspring by a wild male were:

$$
\begin{array}{cccc}
\operatorname{red} \%(6) & \text { white } \sigma^{7}(3) & \text { eosin } \sigma^{7}(2) & \text { eos. verm. } \sigma^{7}(1) \\
119 & 46 & 39 & 18
\end{array}
$$

I had found females of this type before, but had not determined whether the condition might be transmitted, so as to appear in $F_{2}$. Three pairs of $F_{1}$ red female by eosin vermilion brothers gave:

wh.eos. wh.eos.

red $q$ comp. $q$ pink $q$ pink $q$ white $\sigma^{\pi}$ red $\sigma^{\pi}$ yerm. $\sigma^{\pi}$ pink $\sigma^{\pi}$ verm. pink $\sigma^{\pi}$

$\begin{array}{rrrrrrrrr}63 & 50 & 14 & 14 & 69 & 30 & 20 & 10 & 3 \\ 37 & 51 & 32 & 10 & 33 & 36 & 12 & 7 & 2 \\ 65 & 55 & & & 72 & 38 & 19 & & \end{array}$

In each of the first two bottles appeared a pink eyed female splotched with white. One of these was sterile, and the other gave by a pink brother only unsplotched pinks for three generations.

$\begin{array}{ccc} & \text { pink } \% & \text { pink } \sigma^{\top} \\ \mathrm{F}_{3} & 26 & 22 \\ \mathrm{~F}_{6} & 132 & 105 \\ \mathrm{~F}_{5} & 112 & 118\end{array}$

Mosaic forms can be explained by somatic non-disjunctions. 
$P_{1} \quad$ vermilion pink $q$ o eosin vermilion pink $\sigma^{\pi} \sigma^{\pi}$

$F_{1} \quad$ verm. pink 9

heteroz. for $\begin{array}{ccc}\text { eosin } & \text { verm. pinkơ } & \text { eos. verm. pink } \sigma^{7} \\ 307 & 290 & 1\end{array}$

white $\sigma^{7} \sigma^{7}$

$\begin{array}{cccc}P_{1} & \text { pink } \% \text { } \% & & \text { white } \sigma^{7} \sigma^{7} \\ F_{1} & \text { red } \% & \text { red } \sigma^{\pi} & \text { white } \sigma^{7} \\ & 168 & 159 & 2 \\ & 17 & 12 & 4^{*}\end{array}$

* This culture was from a pair.

\begin{tabular}{cccc}
$P_{1}$ & \multicolumn{2}{c}{ pink $\%$ \% } & white verm. pink $\sigma^{7} \sigma^{71}$ \\
$F_{1}$ & $\operatorname{pink} \%$ & pink $\sigma^{7}$ & white (verm. pink) $\sigma^{7}$ \\
& 260 & 231 & 1
\end{tabular}

This one white male I mated to wild females and in $F_{2}$ obtained

$\begin{array}{ccccccc}\operatorname{rcd} 9 & \text { pink } q & \text { white } \sigma^{\top} & \text { red } \sigma^{\top} & \text { verm. } \sigma^{\top} & \text { pink } \sigma^{\top} & \text { verm. pink } \sigma^{\top} \\ 99 & 30 & 64 & 25 & 14 & 13 & 4\end{array}$

This is an extremely close approximation to the result expected on the assumption that the grandfather was vermilion and pink as well as white. Thus, although to sight the vermilion was masked by the white, its reappearance in $F_{2}$ makes another instance where the whole set of sex-linked factors of the father appeared in his exceptional sons in their original combination exactly. The simplest hypothesis is one which assumes a common carrier for all sex-linked factors and the sex differentiator. Just such a carrier is offered by the sex chromosome.

Dexter ('12, p. 190), crossed a yellow female heterozygous for white to a gray white male. There were produced:

$\begin{array}{ccccc}\text { gray red } \% & \text { gray white } \% & \text { yellow red } \sigma^{7} & \text { yellow white } \sigma^{\top} & \text { gray wh. } \sigma^{7} \\ 116 & 116 & 82 & 100 & 2\end{array}$

These gray white males which Dexter was unable to account for in any way, are exactly like their father, as is characteristic of non-disjunctional sons.

When the white eyed mutant first appeared, it was mated to normal red females. In $F_{1}$ there appeared five white males, which Morgan explained as further mutations. It seems to me more likely that these are non-disjunctional males. 
It is possible that some of the anomolous appearances of flies in the cultures of Morgan and Cattell may be explained in this way.

The case referred to by Sturtevant (' $13 \mathrm{~b}$ ) is probably one of non-disjunction coming from miniature white stock.

There are many other cases similar to these, some of which involve other sex-linked factors than those already given.

The sum of all the $F_{1}$ counts derived from single females which were themselves matroclinous is as follows:

$\begin{array}{cccc}\text { matroclinous } \$ & \text { hybrid } q & \text { matroclinous } \sigma^{7} & \text { patroclinous } \sigma^{7} \\ 133 & 2934 & 2677 & 138\end{array}$

This gives the percentage of exceptions as 4.83 , or roughly 5 per cent.

At first glance it would seem that these exceptions are the result of partial sex linkage, that is, that we are dealing with a linkage ratio in which sex is one of the characters. That this is not the case, can be seen from the fact that the results are independent of what particular sex linked factor is used, while in linkage, each different combination has a characteristic value, for instance, the percentage of crossover of white and yellow is about 1.2 per cent, of white and vermilion about 33 per cent, of white and miniature about 35 per cent, and of white and rudimentary about 44 per cent. If the sex differentiator gives 5 per cent of crossover with white it ought to give $33+5$ or $33-5$ with vermilion, $35+5$ or $35-5$ with miniature and, so on. From the fact that I get no such variation depending on the particular factor used, I conclude that this phenomenon is not one of partial sex linkage. It is obvious, however, that nondisjunction forms an alternative hypothesis for all the cases which have been described as partial sex linkage. In some of these cases it is to be preferred, but no decisive choice can be made in any particular case between the two hypotheses, unless, as in Drosophila, there are present more than one sex linked factor.

Morgan and Sturtevant have shown that a certain number of sex linked factors can be arranged in a consistent linear series if the percentage of crossovers be used as the measure of the dis- 
tance between the factors, for example, if $\mathrm{A}$ and $\mathrm{B}$ give 20 per cent of crossover and $A$ and $C$ give 10 then $B$ and $C$ will give approximately ${ }^{5}$ either $20+10$ or $20-10$ as the percentage of crossover. Suppose the experiment gives 10 per cent. Then the order becomes A, B, C, and not A C B. This order when once discovered is found to hold when tested out with any other sex-linked factor. If we know how far a new factor $D$ is from $A$ and $B$ we can predict the percentage of crossover of $C-D$, or if we know its distance from $\mathrm{A}$ and $\mathrm{C}$ we can predict $\mathrm{B}-\mathrm{D}$. At present the series of known sex-linked factors numbers twentyeight. So far as tested, these factors behave as though they occupied loci in a linear series with fixed order and distances, and we believe that this behavior is explicable on the view that the sex chromosome is the material basis of the series.

The bearing of non-disjunction upon this question is that the series of factors which has been shown to be linear, behaves here as though it were a unit. The whole series that the mother bore descends to her matroclinous daughters, and the whole series of the father to his patroclinous sons.

It will have been noticed that in all these cases of non-disjunction, sex behaves in a fashion strictly parallel to that of any sex-linked character. The non-disjunctional eosin or white female transmitted her femaleness to her matroclinous daughters in exactly the same manner that she transmitted her eosin or white eyes. This behavior requires a common vehicle for the transmission of sex and a linear series of sex linked characters, and such a vehicle is furnished by the sex chromosome.

-If we accept the conclusion that the matroclinous daughters are the result of the fertilization of two- $X$ eggs by the no- $X$ spermatozoa, and the patroclinous sons are the result of the fertilization of no- $X$ eggs by one- $X$ spermatozoa, then we must inquire what would result from the fertilization of the two- $X$ eggs by the one- $X$ spermatozoa, and from the fertilization of the no- $X$. eggs by the no- $X$ spermatozoa. In the first case an individual with three, and in the second case with no sex chromo-

${ }^{5}$ Inaccurate for long distances because of double crossing over.

THW JOURNAL OF EXPERIMENTAL ZOZLOGY, VOL. 15, NO. 4 
somes should result. In my experiments I have seen nothing to correspond to the last case, and do not know what to look for, on the dubious assumption that such a fly could live. The three chromosomal form, I believe, would be a female on the assumption that two sex chromosomes bearing the sex differentiator produce a female, and that the extra chromosome would not change this result. If we suppose that the result is a female, what characters will she show? A two- $X$ egg from an eosin female fertilized by a red male would have two eosin factors and the dominant red allelomorph. It seems likely that this female would be red, perhaps diluted somewhat. I have never been able to discover any eye color differences in the large class of red females. Several taken at random, were tested individually with wild males. If one of these females were three chromosomal I expected that there would be two eosin to one red male, and that there would be a preponderance of females, in the ratio of about two females to one male instead of the normal $1: 1$ ratio. The thirty cases tested gave, however, only the regular proportion, 2 red $\&: 1$ red $\sigma^{\pi}: 1$ eosin $\sigma^{\pi}$ Using another and better test, I am about to renew the search for these three- $X$ females.

Although, in Drosophila, there is as yet no direct cytological evidence concerning non-disjunction of chromosomes, in other forms, there is cytological evidence in harmony with what the genetic evidence demands in the present case. The work of Prof. E. B. Wilson ('09) on Metapodius furnishes two such instances. Here the number and size of the chromosomes in each individual is constant, but this number ranges from 21 to 28 , the variation being due to loss of a $Y$-chromosome from the typical 22-chromosome form to give the 21 form, and the addition of one to six supernumerary $Y$-chromosomes to give the higher groups. In the 22-chromosome form, Wilson observed a few second spermatocyte divisions where both $X$ and $Y$ passed to the same pole. The result would be spermatozoa containing respectively twelve and ten chromosomes. Wilson has shown that if these spermatozoa are functional, the whole series of variations follows. The production of spermatozoa with two or with no idiochromosomes 
is directly comparable to the production of two- $X$ and no- $X$ eggs.

Wilson ('10) found an individual of Metapodius in which there were three homologous $m$-chromosomes. He explained this individual in the same manner as the result of the union of an exceptional two- $m$ gamete and the regular one-m gamete.

In the case of Diabrotica, Miss Stevens ('08) found a variation in the number of supernumeraries from zero to four, with inequalities of the distribution in gametogenesis such as Wilson found in Metapodius.

The closest parallel, is perhaps in the case of Ascaris megacephala as reported by Frolowa ('12). Here the $X$ is attached to the end of an autosome, as it perhaps is in Drosophila. However, Frolowa found in exceptional eggs that both sex chromosomes became attached to one member of the pair of autosomes instead of one $X$ to each member. During maturation, there will arise two- $X$ and no- $X$ eggs determined by which autosome of the two, passes out into the polar body. Her diagram for Ascaris fulfils all the requirements for the case of non-disjunction in Drosophila, and, from cytological grounds, she reaches nearly the same conclusion with respect to the fate of the two- $X$ and no- $X$ eggs, as I do from the side of genetics.

The phenomenon of non-disjunction is most readily discovered in any form when the non-disjunction occurs in the sex chromosome, but it might occur with respect to any autosome, where it would only be detected in certain cases. I have a case which may be non-disjunction of the third chromosome of Drosophila, and probably cases of so called "monolepsis" or "faux hybrides" will be found to depend upon this phenomenon. Some forms of parthenogenesis probably differ from non-disjunction only in that the whole number of chromosomes is concerned and not one, or a limited number.

Columbia Univarsity
July 7,1913 


\section{BIBLIOGRAPHY}

Dexter, J. S. 1912 On coupling of certain sex-limited characters in Drosophila. Biol. Bull. vol. 23, no. 3.

Frolowa, Sophia 1912 Idiochromosomen bie Ascaris megalocephala. Archiv f. Zellf. Bd. IX, Heft 1.

Stevens, N. M. 1908 The chromosomes of Diabrotica vittata, Diabrotica soror, and Diabrotica 12-punctata. A contribution to the literature on heterochromosomes and sex determination. Jour. Exp. Zoöl., vol. 5, no. 3.

Sturtevant, A. H. 1913 a The linear arrangement of six sex-linked factors in Drosophila, as shown by their mode of association. Jour. Exp. Zoöl., vol. 14, no. 1 .

$1913 \mathrm{~b}$ The Himalayan rabbit case, with some considerations on multiple allelomorphs. Am. Nat., vol. 47.

$1913 \mathrm{c}$ A third group of linked genes in Drosophila ampelophila. Science, vol. 37 , no. 965 .

WiLson, E. B. 1909 The chromosomes of Metapodius. A contribution to the hypothesis of the genetic continuity of chromosomes. Studies on chromosomes, V. Jour. Exp. Zoöl., vol. 6, no. 2.

1910 A new type of chromosome combination in Metapodius. Studies on chromosomes, VI, Ibid., vol. 9, no. 1. 